\title{
IMPROVED PSI PERFORMANCE FOR LANDSLIDE MONITORING APPLICATIONS
}

\author{
Javier Duro, Rubén Iglesias, Pablo Blanco, Francisco Sánchez and David Albiol \\ Altamira-Information, C/Corsega, 381-387, E-08037, Barcelona (Spain), Email: Javier.duro@altamira-information.com
}

\begin{abstract}
The work proposed in this paper shows advanced Persistent Scatter Interferometry (PSI) processing based on the use of the Non-Local Interferometric SAR (NLInSAR) filtering for improving landslides monitoring applications. The rationale is to enhance the interferometric phase quality, preserving the fullresolution of SAR images, in order to detect both distributed and deterministic scatterers, with the objective of increasing the density of final PSI products. Moreover, since SAR systems are only sensitive to detect displacements in the line-of-sight (LOS) direction, the importance of projecting PSI displacement products along the steepest gradient of the terrain slope is put forward. Two TerraSAR-X data sets acquired in ascending mode corresponding to the period from April to November 2011, and from August to November 2013, respectively, over the area of El Portalet (Central Spanish Pyrenees), are employed for this purpose.
\end{abstract}

\section{INTRODUCTION}

The accurate monitoring of active landslides represents one of the most interesting applications in Differential SAR Interferometry (DInSAR). The characterization of active slopes has been traditionally addressed through the employment of geotechnical techniques, such as inclinometers, extensometers, piezometers, or Differential Global Positioning System (DGPS) networks. Despite providing very accurate information, these in-field devices generally collect scarce measurements in terms of coverage compared with DInSAR products. Moreover, they require the in-situ installation of instrumentation over the slopes, which can be a difficult task when the area of interest is not sufficiently serviced. The improvements in DInSAR and, especially, in Persistent Scatterer Interferometry (PSI) [1-6], have demonstrated some competitive advantages compared with these traditional geotechnical techniques for landslide monitoring applications.

Landslide instabilities typically occur in natural environments largely affected by temporal decorrelation phenomena [7] in which DInSAR performance generally decreases. This fact leads to a poor number of reliable measurement data and compromises the performance of PSI techniques. This issue is especially important in these types of applications where improving the density of persistent scatters as much as possible is a key factor for reaching a better understanding of the extension of the landslide and dynamics.

There are two main classical approaches to detect persistent scatterers. The former is based on exploiting the interferometric coherence information along the multi-temporal set of interferograms. The latter is based on the SAR images' amplitude behavior along the temporal axis through the computation of the amplitude dispersion index (DA). The choice of the pixel selection approach depends on the nature of the area of interest. On the one hand, the coherence stability approach requires performing a multi-look of the interferograms, which allows a significant reduction of the signal-tonoise ratio (SNR), but also leads to a reduction of the spatial resolution. This option is more suited over natural environments with predominance of distributed scatters. Contrarily, the amplitude dispersion approach preserves the full-resolution of SAR images presenting a good performance in the detection of deterministic point-like scatterers, especially over urban environments with predominance of man-made structures.

Hence there is a clear limitation when the PSI processing requires the exploitation of both types of scatterers, distributed and deterministic ones. In this context, different de-noising filtering strategies, typically employed in image processing applications to reduce additive Gaussian noise, have been adapted for InSAR and DInSAR applications, showing an excellent performance for multiplicative speckle noise [6,8-10]. The rationale is to improve the interferometric phase quality, without losing resolution in order to detect both types of scatterers.

The work proposed in this paper shows an advanced PSI processing based on the use of the Non-Local Interferometric SAR (NL-InSAR) filtering technique presented in [10] for landslide monitoring applications. The PSI processing carried out in this work has been addressed employing the SPN technique, the advanced PSI processing chain developed by the company Altamira-Information [4]. This methodology allows the number of looks pixel-by-pixel, to be varied as it depends on the number of similar patches found in a search window around the pixel under analysis, preserving the resolution over point-like scatterers and averaging homogenous regions with predominance of distributed scatterers [10]. In addition, adaptive coherent threshold selection criteria are also employed to select the optimum InSAR pairs to improve the detection of coherent scatterers in long-term stacks of data. The combination of the two solutions is extremely important to improve the final density of measurement points in 
highly decorrelated environments and, therefore, provides more valuable short and long-term solutions over active slopes.

Finally, since SAR systems are only sensitive to the detection of displacements in the line-of-sight (LOS) direction, the importance of projecting final PSI displacement products along the steepest gradient of the terrain slope is put forward.

\section{TEST SITE AND DATA SET}

\subsection{Geological settings of El Portalet}

The test site area selected to evaluate the improvements proposed in this paper corresponds to the landslide of El Portalet, located in the upper part of the Gállego River basin (Central Spanish Pyrenees). This area and surroundings are characterized by a high density of landslides, which have been reactivated due to the constant erosion of the Gállego River as well as human activity [11]. In fact, this is the concrete case of the landslides of El Portalet area, which were reactivated by the excavation work carried out to build a parking area at the bottom of the slope during summer 2004. The area of interest is affected by three main rototranslational landslides (referred to as A-B-C in Fig. 1). The red line represents the contour of a local slide located over the parking area, which shows the higher displacements.

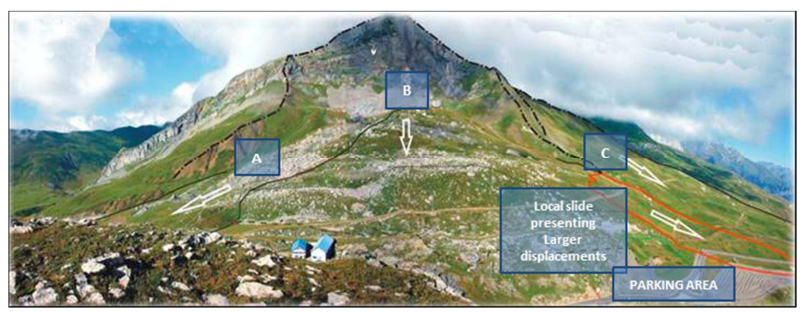

Figure 1. General overview of El Portalet landslides.

El Portalet landslide area is located on a southwestfacing hillside of Petrasos Peak, which is $500 \mathrm{~m}$ long and $700 \mathrm{~m}$ wide and covers $0.35 \mathrm{~km}^{2}$ in about $12^{\circ}$ slope angle [12]. The moving mass also involves intense weathering slates and shales forming high plasticity deposits from the Devonian and Carboniferous age rocks [12]. These geomechanical features are prone to develop the typical morphologies which characterize unstable slopes, where landslides normally occur. Some external features that define typical landslide areas include clearly defined main scarps, hummocky surface topography, high density of surface cracks, streams and pools generated in the landslide limits. The intense rainfalls and the strong topographic gradients intensify the slope mass movement activity in the whole valley.

\subsection{Previous works carried out}

As commented above, the excavation work carried out to build the car park located at the foot of the hillside during summer 2004 provoked a local rupture. This fact has motivated an exhaustive monitoring of the area employing several surveying techniques, such as DGPS, extensometers, inclinometers and Ground-Based SAR (GB-SAR), jointly with extensive geological interpretation works, during the last decade.

In this framework, seven total station measurement campaigns were performed from December 2004 to March 2005. Furthermore, several inclinometric readings were carried out between May and October 2005. Before the breakage of the inclinometers installed, the displacement magnitude and extension of an underground rupture surface were detected and measured in the area. Following the stabilization intervention in the slope, five precise DGPS campaigns were carried out in the area from May 2006 to July 2007 indicating a deceleration process in the slope. At this stage, GB-SAR measurements were planned in order to monitor the car park landslide while the DGPS campaigns continued. Extra DGPS campaigns carried out until October 2011 completed the whole period of measurements from May 2006 to October 2011 [12].

In order to complete these in-field measurements, several advanced PSI studies using space-borne SAR data were carried out. A multi-frequency study with different satellites was performed by AltamiraInformation using C-band data acquired with ERS and ENVISAT satellites (2001-2007), L-band data from ALOS-PALSAR (2007-2011), and X-band data provided by the TerraSAR-X satellite (2008) [13]. Results from these PSI studies reinforced the idea of developing a filtering criterion to increase the measurement point density. Some modeling works have been also carried out in this area. In this context, a recent study based on a $2 \mathrm{D}$ viscoplastic constitutive model [14] has served to validate the extension and the displacement patterns retrieved by means of groundbased and satellite PSI techniques.

\subsection{Data set}

The new generation of X-band space-borne sensors (such as TerraSAR-X and COSMO-SkyMed), allow for a significant improvement in the monitoring capabilities over man-made structures, such as buildings, bridges, railways and highways, but also over natural stable reflectors, such as rocky areas or bare surfaces. This is strongly related with their high spatial resolution, but also with the shorter revisit time, thus improving the temporal and spatial sampling for geo-hazard assessment applications, such as landslide monitoring applications.

To continue with the monitoring of El Portalet carried out during the last years, two TerraSAR-X data sets acquired in ascending mode, with the objective of maximizing the sensor sensitivity to the displacement (as it will be explained in Section 5), have been selected. The fast temporal decorrelation phenomenon present at X-band over this type of natural environment 
represents a perfect test site to demonstrate the benefits of using NL-InSAR for PSI purposes. The first data set is composed of 13 TerraSAR-X images acquired during the period corresponding from April 2011 to November 2011. The second one is composed of 8 TerraSAR-X images acquired during the period from August to November 2013.

In order to improve the PSI performance 7 Artificial Corner Reflectors (ACR) for radar satellite were installed in September 2010. In 2013, an intensification of the ACR network, adding 11 new ACRs, was carried out in the main slope of El Portalet. Finally, an Automatic Inclinometer System (AIS) [15-17] was installed in a new borehole in August 2013. DGPS over the new ACRs installed and over other interesting points of the landslide have been collected to date.

\section{THE SPN TECHNIQUE}

PSI is an evolution of DInSAR that allows a reliable estimation of the displacement component $\varphi_{\text {displ }}$ present in differential interferograms phase $\varphi_{\text {dif }}$ to be performed making use of a multi-temporal stack of SAR images in order to overcome the limitations of classical DInSAR. The use of a stack of interferograms allows for the estimation of the different components present in the $\varphi_{\text {dif }}$, expression:

$$
\begin{aligned}
\varphi_{\text {dif }} & =\varphi_{\varepsilon D E M}+\varphi_{\text {displ }}+\varphi_{A P S}+\varphi_{\text {noise }} \\
& =\frac{4 \pi}{\lambda} \frac{B_{n}}{R \sin \theta_{\text {inc }}} \varepsilon_{D E M}+\frac{4 \pi}{\lambda} \rho+\varphi_{A P S}+\varphi_{\text {noise }}
\end{aligned}
$$

where $\varphi_{\varepsilon D E M}$ refers to the phase term corresponding to the height inaccuracies of the DEM employed in the differential interferogram generation $\left(\varepsilon_{D E M}\right), \rho$ accounts for the LOS displacement, $\varphi_{A P S}$ accounts for the atmospheric artifacts phase term and $\varphi_{\text {noise }}$ [7] accounts for the phase component containing the decorrelation terms (such as the spatial and temporal ones) present in the differential interferograms.

Several advanced PSI processing chains have been developed over the last decade in order to estimate surface displacements with extremely high accuracy [15]. The work presented in this paper has been carried out using the SPN, which stands for the advanced PSI technique developed and developing by the company Altamira-Information since 2002 [4].

In order to perform precise estimations, only those pixels that are not affected by decorrelation are first detected. The reliability of any PSI processing technique is compromised by the phase quality of the differential interferograms available. Mainly due to temporal decorrelation phenomena [7], only a limited number of them fulfill the phase quality requirements to obtain reliable displacement map estimations. For this reason, prior to the application of any PSI technique, an adequate selection of reliable high-quality pixel candidates is mandatory, i.e., the so-called persistent scatterers $[1,3]$. The point density depends on the land cover (urban, vegetated, arid, etc.), but values of several thousands of measurement pixels per square kilometer are typically obtained. As will be shown in the following Section, the number of high-quality pixels for the SPN processing can be improved by applying advanced adaptive filtering techniques.

As seen in Eq.1 the evolution of the differential interferometric phase along the temporal stack is directly related to the ground displacement produced, but there are other artifacts that may compromise the reliability of the displacement estimation process. On the one hand, as seen above, the real height of the stable points considered for the PSI processing can differ from what it is indicated by the DEM depending on its accuracy. This height error has a negative impact for PSI purposes and must be accordingly estimated and compensated for. On the other hand, the impact of the APS must be also considered. The variability of the atmospheric artifacts during the temporal axis also affects the differential phase. Due to the atmospheric changing conditions, the delay that occurs when an electromagnetic wave passes through the different layers of the atmosphere is variable from one image to another, but also within the same image depending on the location. These effects must be estimated and compensated for in order to obtain a precise measurement of the actual displacement. The common approach to compensate APS consists of taking advantage of their particular temporal and spatial frequency behavior. This problem is typically addressed through a low pass filtering process [7], which also allows the cancelation of the different offsets between the differential interferograms available.

The SPN consists of a pixel-by-pixel process to obtain the linear and non-linear components of displacement, but also the topographic error and the APS. The first block of the SPN is based on adjusting a linear modelbased solution to the differential interferograms available, taking into account the different terms of the differential phase equation seen in Eq. (1). This estimation is performed pixel-by-pixel without the employment of any spatial interpolation. As a result, a reliable mean annual deformation rate and a topographic DEM error are obtained for the persistent scatterers available. At this stage, the so-called model coherence [3] is defined in order to measure the quality of the solutions. The model coherence ranges in the interval $[0,1]$, reaching high values when the linear model perfectly fits the data. Otherwise, it tends towards to zero when the model adjustment is poor. With this function it is possible to discard noisy pixels due to a bad adjustment.

Once the linear estimation step is finished, the SPN is able to provide time series related with the temporal evolution of the total displacement. For this purpose, the linear model obtained previously, and the APS estimated through spatiotemporal filtering techniques, 
are removed from the original differential interferograms leading to the so-called phase residues, which will only contain the non-linear component of displacement. The rationale is to re-order these differential residues along the temporal axis to obtain the absolute non-linear displacement per image to subsequently add them to the linear model estimated in the previous step. Time series are typically employed to identify non-linear patterns and identify possible accelerations. The accuracy of the process is roughly 2$5 \mathrm{~mm}$. This value depends on the satellite, the number of images and on the quality of the measurement points. Time series can be optionally filtered in order to reduce the possible remaining noise and ease the interpretation of the final results.

\section{NL-INSAR FILTERING}

NL-InSAR filtering consists of a probabilistic similarity criterion based on both the intensities and the interferometric phases that surround two given patches [10]. Nonlocal filter uses the concept of local redundancy for estimating, pixel-by-pixel, the scene components preserving the resolution of the image. This methodology allows for the consideration of pixels that are far apart rather than restricting the similar pixels that belong to a local neighborhood, such as boxcar filtering (see Fig. 2).

One pixel is assumed to come from the same statistical population as the given pixel if the patches that surround both pixels present a high similarity measure [10]. The filtered pixel is obtained by a weighted combination of pixel values, which can be far apart inside the search window (see Fig. 2(c)). The weights are computed using the maximum likelihood estimator (WMLE) and are iteratively refined by comparing patches of previous estimates and patches of the noisy image [10].

Fig.3 illustrates the benefits of applying NL-InSAR filtering to improve the quality of differential interferograms. Fig.3(a) shows the original interferogram at full-resolution, while Fig.3(b)-(c) shows the resulting differential interferogram after applying a $5 \times 5$ boxcar window and a NL-InSAR filtering (using a patch window of 5 pixels and a search window of 20 pixels), respectively. Notice the quality of NL-InSAR filtering, which smooths the noise over the homogeneous regions while maintaining the resolution over the deterministic point-like scatterers.

Under this approach the number of looks varies from one pixel to another as it depends on the number of similar patches found in the search window around the pixel under analysis. The sizes of the patch and search window are set as a trade-off for having one look in the ACRs present in the scene, but also to ensure enough noise reduction over the homogeneous areas. This fact is illustrated in Fig.4, which shows the different number of looks obtained in 3 different land covers. Notice how

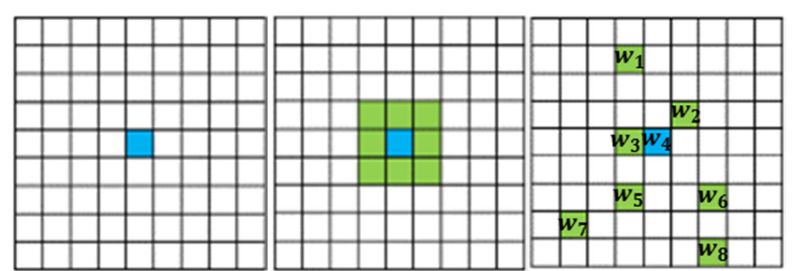

(a)

(b)

(c)

Figure 2. Comparison between (a) full-resolution, (b) boxcar, and (c) adaptive NL-InSAR filtering.

the number of looks is larger over the bare surfaces of the area studied, while the ACR has only 1 look, as it should be, since it is a deterministic point-like scatterer. Once the interferograms are filtered, PSI processing is totally equivalent, being no different with respect to the classical approaches.

\section{DOWN-SLOPE HYPOTHESIS}

SAR systems only have sensitivity in the LOS direction and hence, the displacement maps obtained by means of PSI techniques are generally referred to the slant-range plane. For the current polar-orbiting SAR space-borne sensors, the look direction is either east or west, for ascending or descending orbits, respectively. For this reason, SAR sensors are only sensitive to movements along slopes facing either east or west, and almost insensitive to movements in north or south directions. This aspect should be taken into account when planning the SAR acquisitions.

In the context of landslide applications, the more realistic assumption about the displacement direction is based on considering that it is produced along the steepest gradient of the slope. Computing the percentage of measured displacement in the LOS direction is mandatory in order to ensure reliable results.

For the study case presented in this paper the ascending mode resulted more suited since the projection factor between the displacement and LOS directions was lower compared with the one provided by the descending mode. The ascending mode was therefore finally selected in order to maximize the percentage of displacement detected by the space-borne sensor.

Another factor to take into account in these types of applications is related with the interpretation of PSI results. Over flat areas this fact does not represent any difficulty since the displacements are typically addressed as negative departing from the sensor (for subsidence phenomena) and positive approaching the radar (for uplift phenomena). On the contrary, when facing areas with steep topography, as in the present case, the interpretation of the final results becomes more complex. If the slope of the mountain is facing the satellite, any land movement along the down-slope direction will be towards the satellite. Contrarily, the same displacement will be detected as moving away from the SAR sensor if the slope is opposite.

Finally, due to this particular acquisition geometry, 


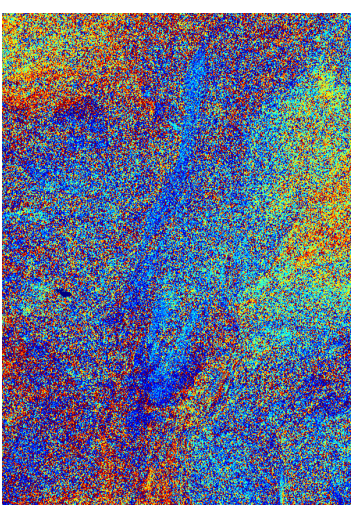

(a)

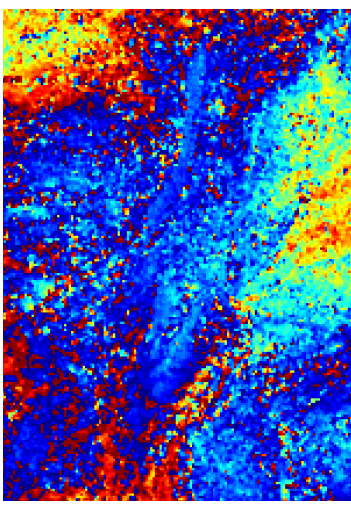

(b)

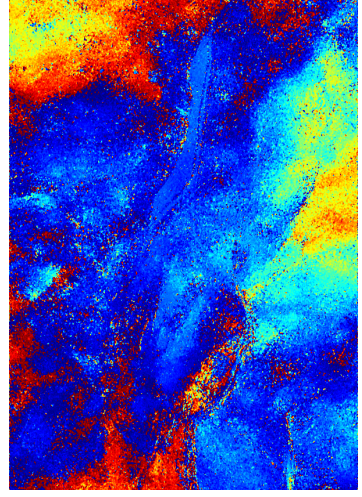

(c)

Fig.3. (a) Full-resolution interferogram. Filtered interferogram after applying (b) a $5 \times 5$ boxcar window and (c) adaptive NL-InSAR filtering.

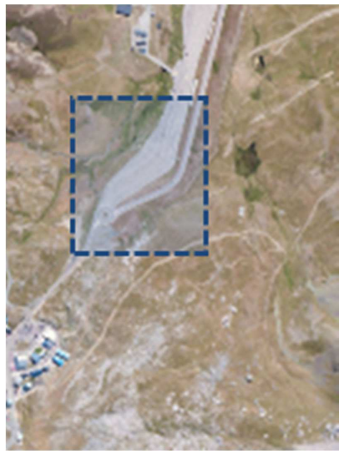

(a)

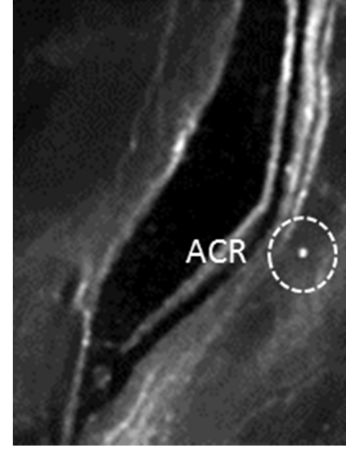

(b)

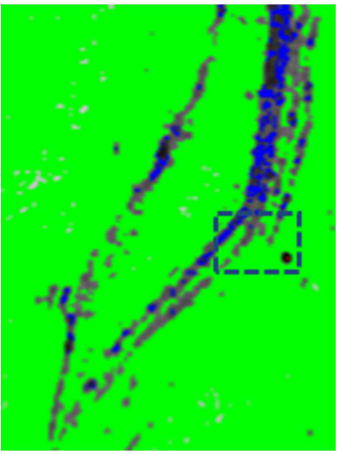

(c)

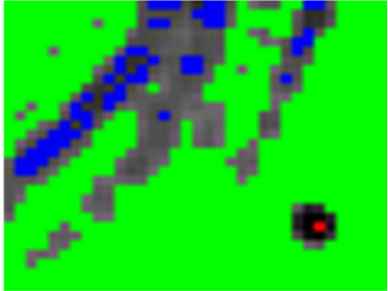

- 1 look: ACR

- 70 -100 looks: Road

- 200 -300 looks: Bare Soil

(d)

Fig.4. Different number of looks obtained in 3 different land covers. (a) Optical image corresponding to the parking area of El Portalet. (a) SAR Reflectivity image of the area indicated with a blue square in (a). The white circle indicates the location of an ACR. (c) Different number of looks obtained over the bare soil, the road and the ACR available within the selected area. (d) Zoom of the area indicated with a blue square in (c).

SAR geometrical distortions should be also taken into account when planning the acquisitions and, furthermore, in the final interpretation of the displacement results. These are the foreshortening, the layover and the shadowing [7].

Under the argument described above, the measured displacements in the LOS direction are rarely the real ones, but a projection of them. Following a vector notation, both velocities may be expressed mathematically as follows

$$
\begin{aligned}
& \mathbf{V}_{\text {LOS }}=\left|\mathbf{V}_{\text {LOS }}\right| \cdot \hat{\mathbf{l}}_{\text {LOS }} \\
& \mathbf{V}_{\text {Ground }}=\left|\mathbf{V}_{\text {Ground }}\right| \cdot \hat{\mathbf{l}}_{\text {Ground }}
\end{aligned}
$$

where $\mathbf{V}_{\text {Ground }}$ accounts for the ground velocity vector, i.e, the real ground displacement, and $\mathbf{V}_{\mathbf{L O S}}$ the measured velocity vector, which is a projection of the previous one in the LOS direction. The magnitudes $\left|\mathbf{V}_{\mathbf{L O S}}\right|$ and $\left|\mathbf{V}_{\text {Ground }}\right|$ account for the magnitude of the displacements, and the unitary vectors $\hat{\mathbf{I}}_{\text {LOS }}$ and $\hat{\mathbf{I}}_{\text {GROUND }}$ are related with the displacement directions. Since the former is a projection of the latter the intensity of both vectors may be related through a scalar product

$$
\left|\mathbf{V}_{\text {Los }}\right|=\left|\mathbf{V}_{\text {Ground }}\right| \cdot \cos (\alpha)
$$

where

$$
\cos (\alpha)=\hat{\mathbf{l}}_{\text {LOS }} \cdot \hat{\mathbf{l}}_{\text {Ground }}
$$

being $\alpha$ the angle formed by both unitary vectors.

When the ground displacements produced over the affected area are expected to be in the vertical direction over flat areas, as typically occur with subsidence or uplift phenomena in urban scenarios, $\alpha$ directly becomes the incidence angle. Contrarily, in landslide monitoring applications the problem becomes more complex since the real ground displacement of each spatial point has a topographic dependence related with the local slope in each position. As seen above, the more realistic assumption of the displacement direction with no a priori knowledge consists of considering that the displacement is produced along the steepest gradient of the terrain slope. This information may be directly derived employing an accurate DEM of the illuminated 
area, and the angle $\alpha$ may hence be obtained through Eq.4. Fig. 5 shows the acquisition geometry under the down-slope displacement assumption described in this Section.

Once the displacement vectors are accordingly projected they can be geocoded in map coordinates and be visualized using a virtual globe viewer or a Geographical Information System (GIS) software.

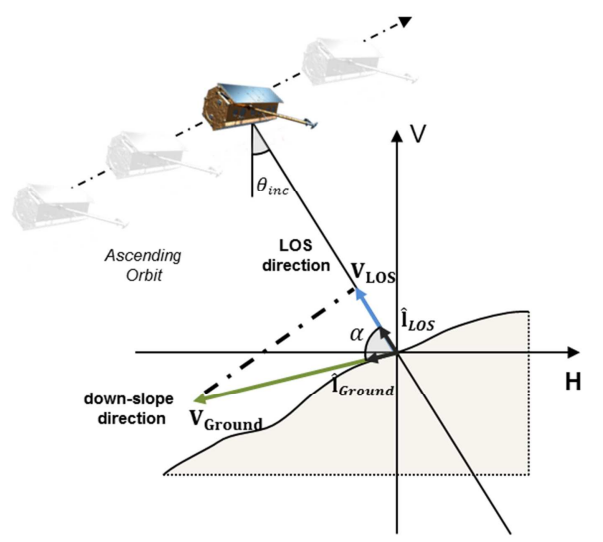

Figure 5. Acquisition geometry under down-slope displacement assumption.

\section{RESULTS AND DISCUSSION}

This Section presents the final accumulated displacement maps over the area of El Portalet by means of the NL-InSAR filtering technique presented in Section 4. Furthermore, the importance of projecting down-slope the final PSI results, as stated in Section 5, is also put forward.

Fig.6 shows a comparison of accumulated displacements in the LOS corresponding to the 2013 TerraSARX data set using the full-resolution DA approach (see Fig.6a), the coherence stability approach using a 5x5 boxcar window (see Fig.6b), and after applying the NL-InSAR filtering technique proposed in Section 4 (see Fig.6c). To ensure a fair comparison, equivalent thresholds over the three methodologies have been established in order to provide the same requirements in terms of phase standard deviation. As expected, all approaches retrieve almost identical displacement trends except for the increase in pixels' density. Specifically, PSI results based on NL-InSAR filtering show an outstanding improvement (roughly a threefold increase) in the detection of persistent scatterers (bare soil, roads, rocks) compared with classical PSI approaches. As seen in Section 4, this advanced adaptive filtering technique allows the joint detection and exploration of point-like and distributed scatterers, thus benefiting final PSI results. It must be pointed out that the availability of ACRs in the area of interest, presented in Section 2, has allowed the maximization of the filter response.
The increase in terms of pixel density showed by the NL-InSAR approach, represents a key factor in the characterization of the landslides available in the area of study. This enhancement allows for the increase in the density of measurements allowing an improvement in determining the extension and dynamics of the landslide.

As stated in Section 5, prior to the interpretation of the displacements results retrieved, PSI products should be projected assuming a down-slope displacement model. Fig.7 shows the final accumulated down-slope displacement results over the area of El Portalet from the two TerraSAR-X data sets available. These results reveal several slope instabilities occurred in El Portalet area during the monitoring period. Notice that the results corresponding to both periods are in concordance since they show similar displacement patterns. As expected, some instabilities may be noticed in the A, B, and $\mathrm{C}$ landslide areas described in Fig.1. Main displacement points up to $30 \mathrm{~cm}$ (from April to November 2011) and up to $10 \mathrm{~cm}$ (from August to November 2013) may be identified at the toe of landslide B (see Fig.8). This region corresponds to the local slide, which is occurring over the parking area. As commented in Section 2, this region is exhibiting the larger displacements of the area during the last years (red contour area represented in Fig.1), however, local and distributed displacement patterns may also be appreciated in landslides areas $\mathrm{A}$ and $\mathrm{C}$ for both periods. These are clearly identified after applying the downslope projection factor.

These conclusions are coherent with the previous results obtained through the in-filed campaigns and DInSAR results presented in [11-13].

Finally, the displacement maps obtained during 2011 show a high agreement with the conclusions extracted from the DGPS campaigns presented in Section 2, as illustrated in Fig.8.

This figure shows a comparison between the downslope PSI results (points) presented in Fig.7 and the displacements vectors retrieved through the DGPS campaigns carried out during 2011 (red arrows) in the parking area. A good correlation in terms of spatial extension and magnitudes may be appreciated, thus validating the down-slope displacement assumption proposed in the paper. Furthermore, notice how the location of main scarps of the landslide area, defined by geomorphological interpretation (black line and orange polygon in Fig.8), are in agreement with the PSI displacements detected. 


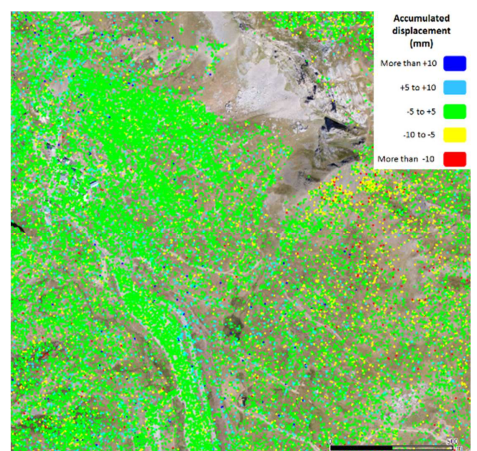

(a)

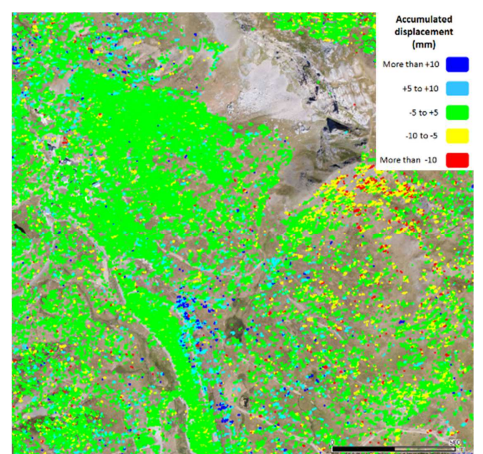

(b)

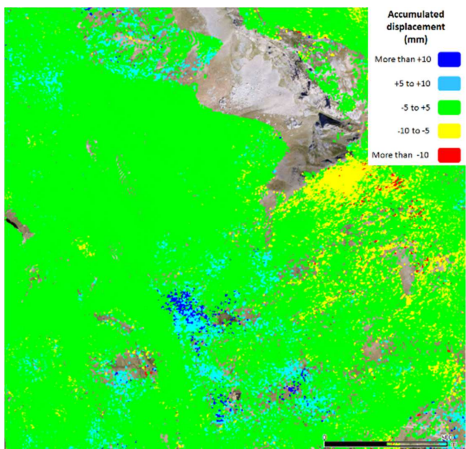

(c)

Figure 6. PSI Displacement results in the LOS direction using a (a) full-resolution PSI processing, after applying (b) $5 \times 5$ boxcar window, and after applying (c) NL-InSAR. Results correspond to the 2013 data set described in Section 2.

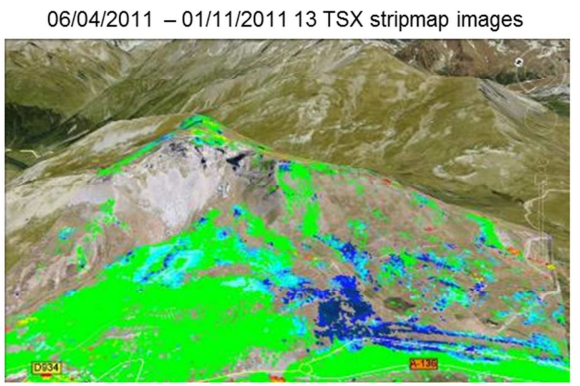

(a)

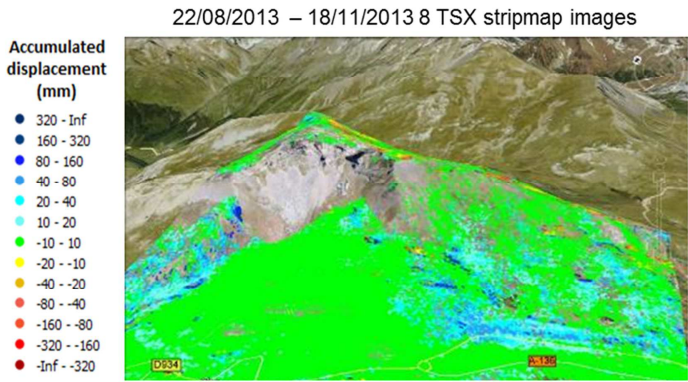

(b)

Figure 7. NL-InSAR down-slope accumulated displacement results over the area of El Portalet from the 2011 and 2013 TerraSAR-X data sets presented in Section 2.

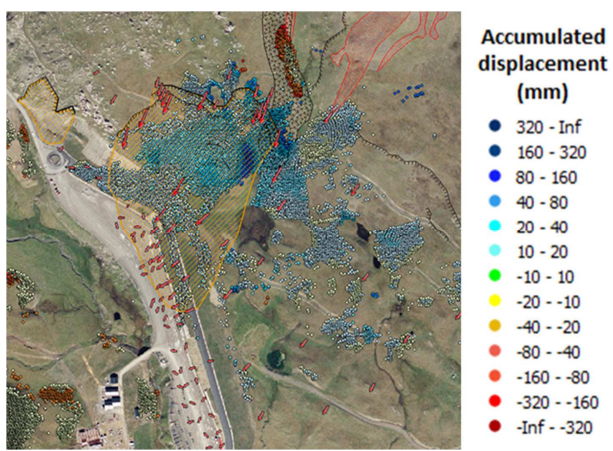

Figure 8. Comparison between the down-slope accumulated PSI results (points) presented in Figure 7 and the displacements vectors retrieved through the DGPS campaigns carried out during 2011 (red arrows) in the parking area.

\section{CONCLUSIONS}

In this paper advanced PSI improvements to enhance the displacement retrieval process over natural environments with different land covers have been presented. On the one hand, the employment of the NLInSAR filtering technique presented in [10] has been proposed in order to enhance the number of reliable high-quality pixels and its quality during the PSI processing. In this framework, a comparison with the so-called classical approaches has been proposed. PSI results by means of NL-InSAR have demonstrated the improvement of both the density and quality of displacement estimations. This is a key factor in landslide monitoring applications, which normally occur in natural environments largely affected by temporal decorrelation phenomena, which are typically characterized by a poor number of persistent scatterers detected. On the other hand, the application of a downslope displacement assumption has been proposed to achieve a correct understanding of the displacements.

The final displacement results obtained over two TerraSAR-X data sets, have presented similar displacement patterns, showing a high level of agreement with the previous field and PSI results presented over the area of interest. Finally, DGPS measurements coinciding with the data set corresponding to 2011 have been presented in order to validate the final PSI displacement results obtained.

\section{ACKNOWLEDGEMENTS}

This research work has been funded by the European Union Seventh Framework Programme (FP7/20072013) in the framework of the LAMPRE project (Grant Agreement 312384). The authors would like to thank 
Dr. Gerardo Herrera, Dr. Juan Carlos García Davalillo and Dr. José Antonio Fernández Merodo from the Geohazards InSAR Laboratory and Modelling Group of the Instituto Geológico y Minero de España, Madrid (Spain), and Dr. Paolo Allasia, Dr. Piernicola Lollino and Dr. Andrea Manconi from the Istituto di Ricerca per la Protezione Idrogeologica, Consiglio Nazionale delle Ricerche, Torino (Italy), for the field instrumentation campaigns carried out and for their helpful discussions during the final displacement results interpretation.

\section{REFERENCES}

1. Ferretti, A., Prati, C., and Rocca, F. (2001). Permanent Scatterers in SAR interferometry. IEEE Transactions on Geoscience and Remote Sensing, 39 (1), 8-20.

2. Berardino, P., Fornaro, G., Lanari, R., and Sansosti, E. (2002). A new algorithm for surface deformation monitoring based on small baseline differential SAR interferograms. IEEE Transactions on Geoscience and Remote Sensing, 40 (11), 2375-2383.

3. Mora, O., Mallorqui, J., and Broquetas, A. (2003). Linear and nonlinear terrain deformation maps from a reduced set of interferometric SAR images. IEEE Transactions on Geoscience and Remote Sensing, 41 (10), 2243-2253.

4. Arnaud, A., Adam, N., Hanssen, R., Inglada, J., Duro, J., Closa, J., and Eineder, M. (2003). ASAR ERS interferometric phase continuity. IEEE International Geoscience and Remote Sensing Symposium, 2, 1133-1135.

5. Blanco-Sánchez, P., Mallorquí, J. J., Duque, S., and Monells, D. (2008). The Coherent Pixels Technique (CPT): An Advanced DInSAR Technique for Nonlinear Deformation Monitoring. Pure and Applied Geophysics, 165 (6), 1167-1193.

6. Ferretti, A., Fumagalli, A., Novali, F., Prati, C., Rocca, F., and Rucci, A. (2011). A New Algorithm for Processing Interferometric DataStacks: SqueeSAR. IEEE Transactions on Geoscience and Remote Sensing, 49 (9), 34603470 .

7. Hanssen, R., Radar interferometry (2001). Data interpretation and error analysis, kluwer aca ed.

8. Deledalle, C.-A., Denis, L., and Tupin, F. (2009). Iterative weighted maximum likelihood denoising with probabilistic patch-based weights. IEEE transactions on image processing. 18 (12), 266172

9. Alonso-González, A., López-Martínez, C., Member, S., and Salembier, P. (2012). Filtering and
Segmentation of Polarimetric SAR Data Based on Binary Partition Trees. IEEE Transactions on Geoscience and Remote Sensing, 50 (2), 593-605.

10. Deledalle, C.-A., Denis, L., and Tupin, F., (2011). NL-InSAR: Nonlocal Interferogram Estimation. IEEE Transactions on Geoscience and Remote Sensing, 49 (4), 1441-1452.

11. García-Ruiz, J.M.and Chueca, J. and Julin, A. (2004). Los Movimientos en Masa del Alto Gallego. Geografía Física de Aragón. Aspectos generales y temticos, 142-152.

12. Herrera, G., Fernandez-Merodo, J., Mulas, J., Pastor, M., Luzi, G., and Monserrat, O. (2009). A landslide forecasting model using ground based SAR data: The Portalet case study. Engineering Geology, 105 (3-4), 220-230.

13. Cooksley, G., Sanchez, F., Sabater, J., and Banwell, M. (2011). Ground motion detection using radar satellites in areas prone to landslides: El Portalet landslide and Valle de Tena, Spain. 11th International Symposium on Landslides and 2nd North American Symposium on Landslides, 2 (2), 1339-1344.

14. Fernández-Merodo, J. a., García-Davalillo, J. C., Herrera, G., Mira, P., and Pastor, M. (2012). 2D viscoplastic finite element modeling of slow landslides: the Portalet case study (Spain). Landslides, 11 (1) , 29-42.

15. Manconi, A. and Giordan, D. (2012). Surface displacements following the Mw 6.3 L'Aquila earthquake: One year of continuous monitoring via Robotized Total Station. Italian Journal of Geosciences, 131 (3), 403-409.

16. Allasia, P., Manconi, A., Giordan, D., Baldo, M., and Lollino, G. (2013). ADVICE: A new approach for near-realtime monitoring of surface displacements in landslide hazard scenarios. Sensors, 13 (7), 285-302.

17. Lollino, G., Arattano, M., Allasia, P., and Giordan, D. (2006). Time response of a landslide to meteorological events. Natural Hazards and Earth System Sciences, 6, 179-184. 